\title{
Editorial
}

\section{EL ENCUENTRO PEDAGÓGICO}

La pedagogía considerada como parte de la construcción social de un contexto y un tiempo, invita a pensar en nuestro país, en el ciudadano que se forma en nuestras escuelas; disciplinado, controlado, religioso, hombre de progreso, racional, activo, autónomo o crítico. Cada uno de estos calificativos, sugiere formas diferentes de relación entre maestros y estudiantes. El instructor, el instruido, el dueño del saber y el aprendiz, el aplicador de teorías y el alumno repetidor de saberes, el educador y el educando, cada cual con su historia, sus reglas, principios y métodos, asiste al encuentro diario con el otro con la intención de controlar el momento, de hacer lo planeado sin embargo, siempre se escapa algo o alguien de los límites que construimos, de la idea que elaboramos del otro, a pesar de querer ignorar que no existe el incontrolable y mucho menos la clave para atrapar algo o alguien para siempre, ciertamente se nos presenta insolente, atrevido jugando con sus múltiples caras, y nosotros en el intento por comprender lo que pasa, nos confunde la velocidad y el movimiento, dando la impresión que es imposible verlo desde el lugar que indicaría una teoría cualquiera.

Como lo menciona Pineau (2001), la pedagogía ha venido reduciéndose al campo escolar, organizada en el siglo XX a partir de la cadena pedagogía- escuela-currículo, esto implica el triunfo de la "racionalidad técnica" a la que debe acoplarse en primera instancia el docente, adoptando el método correcto para disciplinar, controlar y evaluar a sus alumnos y al final convertirse en un técnico de la educación que dará ejemplo de conducta a seguir dentro y fuera de la escuela. El alumno por su parte es objeto de un proceso de normalización que conduce a caracterizar su comportamiento, sus rutinas, el ordenamiento de los espacios, el disciplinamiento reflejado en la disposición de los cuerpos, la asistencia diaria y obligatoria; con todo esto, se pretende garantizar un mecanismo de control social que dará paso a la idea de progreso.

Esta forma de ver la escuela como ordenadora de saberes que le otorga toda la confiabilidad a los métodos, la ciencia y las diversas disciplinas, se ha quedado corta frente a la velocidad con que circula la información. El presente siglo exige transformaciones, acordes con la vida, el movimiento y la tecnología que da otra disposición a los contextos sociales y educativos, los encuentros ocurren en dimensiones de espacio y tiempo real o virtual, la inmediatez se hace presente en los sistemas de información, creando nuevas posibilidades de acción en la lógica global de nuestros tiempos y las mentes de niños, jóvenes y maestros. 
Los maestros y estudiantes del siglo XXI comprometidos con la transformación social, tienen la oportunidad de indagar por la estructura de la escuela, reconocer los nexos que la componen, además, darse cuenta del papel que cada uno cumple en la urgencia de comunicarse para darle valor a sus experiencias.

¿Qué valor tiene el saber pedagógico y las experiencias si no existe la comunicación en el aula? "La pregunta por la pedagogía hoy es la pregunta por el acontecimiento pedagógico y por el pedagogo que habla de él y el maestro que hace experiencia de él, es decir, la pedagogía es del sujeto. En este sentido la pedagogía se convierte en una práctica que se construye en las interacciones, los saberes y las experiencias" [...] (Gómez y Pulido 2016 p 8) La acción de enseñar y aprender, puede ser criticada, cuestionada, transformada por entes reguladores nacionales e internacionales, pero es en la cotidianidad de la vida escolar donde se tejen las relaciones haciendo posible la comunicación que soporta la potencia de una construcción personal y social, un lugar para conversar, para dejar que ocurran acuerdosn y desacuerdos comprendiendo que se manifiestan en la medida que se relacionan con lo que nos pasa.

De acuerdo con lo anterior, cabe señalar que la escuela sigue siendo de cualquier forma un lugar de encuentro donde acontece la vida y por esto mismo se genera permanentemente la fuerza que la mantiene, una de las pocas instituciones en la que seguimos creyendo en Colombia, tal vez, porque no perdemos la esperanza de encontrar caminos que acompañen la construcción de seres humanos transformadores de la sociedad que conocemos. Es necesario reconocer que no basta con la fuerza, no basta con simplemente creer en su existencia, es inaplazable revisar métodos, técnicas, didácticas, teorías, formas de ser $\mathrm{y}$ actuar, elementos que se ponen en juego cada vez que tenemos la oportunidad de pensarnos, de encontrarnos con cuestionamientos y necesidades en un contexto social y un momento histórico.

Tener conciencia de la responsabilidad del encuentro pedagógico, es darse cuenta del otro -estudiante o maestrode su experiencia, sus prácticas, ideas y conceptos construidos en la vida cotidiana que cobran importancia siempre y cuando tengan un espacio en las discusiones del aula. La Maestría en Educación de la UPTC, en su dinámica de formación, ha tenido la oportunidad de encontrarse con más de 400 maestros y sus experiencias, reconociendo que hace falta conversar sobre lo que nos pasa en la escuela y en la universidad Los maestros convertidos en estudiantes, han podido volver a soñar con la escuela más allá de los límites impuestos por las disciplinas, han hecho un alto para dar cabida a la manifestación de intereses, preocupaciones, debilidades, puestas en miradas, imágenes, palabras que fueron mostrando el valor de los acontecimientos en diversas instituciones educativas del departamento, los maestros, desde el dominio de las disciplinas tenían mucho que contar y a la vez escuchar a otros y pensar que a partir de esos encuentros se podía abordar un mismo problema desde las distintas áreas, entonces fue obligatorio discutir, analizar las divergencias y puntos en común para presentar a los lectores el fascículo 20 de la revista Educación y Ciencia. Se publican resultados de investigaciones 
desarrolladas a partir de proyectos pedagógicos que muestran los sentires, saberes y experiencias de maestros de diferentes contextos en escuelas rurales y urbanas, principalmente del departamento de Boyacá.

La revista se presenta en tres secciones o cuadernos, denominados Paideia, Signos y Babelia, de esta forma se agrupan dando un sentido especial de la siguiente manera:

Paideia: se sitúa en una posición analítica y prospectiva para abordar el estudio de investigación en educación y pedagogía. Los debates y las tendencias pedagógicas dejan ver, en primer lugar, el artículo titulado $(\mathrm{R} e)$ significación de la práctica docente: reflexiones acerca del quehacer del maestro, de los investigadores Leonor Itsmenia Granados Becerra,Luis Eduardo Martínez Méndez de la Institución Juan José rondón de Soatá y Sonia Lucía Romero Alfonso de la Universidad Pedagógica y Tecnológica de Colombia, proponen reflexionar sobre la práctica docente de los profesores afrontando cuestionamientos de manera crítica, sobre su quehacer, con el objetivo de (re)significararla, al materializar estrategias hibridas buscando dinámicas respecto del componente didáctico en beneficio del proceso de aprendizaje de los estudiantes. El segundo texto, de las profesoras Yurany Barrera Cárdenas y Rosalba Cristancho Saavedra de la Institución educativa Politécnico Álvaro González Santana, titulado Desarrollo de la competencia de indagación en ciencias naturales, realiza una reflexión que contribuye en la formación del estudiante incrementando el trabajo colaborativo, la responsabilidad y la autoformación.
Los profesores Carlos Julio Vivas Silva, Zulma Lucía Murillo Chiquillo de la Institución Juan José Rondón Tasco, y Jaime Ricardo Cristancho Chinome de la Universidad Pedagógica y Tecnológica de Colombia, en su artículo Estrategia didáctica para el aprendizaje de las tablas de multiplicar en escuela nueva, ven la necesidad de fortalecer procesos multiplicativos y favorecer el pensamiento numérico en la formación integral del educando, contribuyendo a desarrollar habilidades que respondan con eficiencia en la solucionar problemas matemáticos de la vida cotidiana. La siguiente investigación, titulada Elcuento como estrategiapedagógicapara desarrollar la indagación en Ciencias Naturales realizada por la profesora Carmen Estela castillo de la Institución Educativa Agropecuaria el Escobal de Ramiriquí, hace énfasis en la comprensión y construcción de conceptos, capacidad de formulación de preguntas y uso de palabras científicas, para mejorar la competencia de indagación en ciencias naturales, obteniendo como resultado el fortalecimiento de competencias comunicativas, base para la construcción del conocimiento.

La investigación titulada Movilización del concepto ambiente en estudiantes de grado sexto, fue realizada por los profesores: Jenny Natalia Fonseca Rincón de la Institución Educativa Enrique Olaya Herrera y Ricardo Molano Carrera de la Universidad Pedagógica y Tecnológica de Colombia, quienes presentan un estudio con estudiantes de grado sexto ratificando la necesidad de contribuir con una propuesta pedagógica que permita comprender la concepción de ambiente desde una visión sistémica. Los investigadores Alfonso Jiménez Espinosa de la Universidad Pedagógica 
y Tecnológica de Colombia, Laura Emily Parra Quemba y Hollman Dubán Camacho Reyes de la Institución Educativa Antonio Nariño, presentan resultados parciales del proyecto Enseñanza y aprendizaje en resolución de problemas: productos notables, encontrando que, a partir del análisis de tres textos escolares y un cuestionario aplicado a 191 estudiantes, se hace la configuración epistémica y cognitiva del Enfoque Ontosemiótico. Los componentes de idoneidad epistémica, cognitiva y afectiva del enfoque dan relevancia al grado de representatividad del significado institucional, a la apropiación de los conocimientos y al uso de medios tecnológicos.

Signos: el cuaderno Expresiones simbólicas que comunican, diversifican y embellecen el mundo desde el plano de la Lingüística permite agrupar un número más de artículos:

En primer lugar, la investigación titulada El cine, una estrategia para desarrollar babilidades del pensamiento critico en sociales, presentada por las profesoras Sandra Liliana Morantes Cepeda y Yasmin Gordillo Ávila de la Institución Educativa Politécnico Álvaro González Santana, muestra la recordación, contextualización y el fortalecimiento de la expresión escrita y oral sobre temas de Ciencias Sociales en los estudiantes de primero y segundo. El celular: dispositivo tecnológico para el desarrollo de las competencias comunicativas, es una investigación presentada por las profesoras Diana carolina Pineda Díaz, Ximena Gisele Rivera Guío de la Institución educativa Técnica de Nobsa, y Sandra Milena Murcia Pardo de la Universidad Pedagógica y Tecnológica de Colombia, cuyo objetivo fue identificar cómo el uso del dispositivo móvil incide en la mejora de la competencia comunicativa oral de los estudiantes de grado noveno, y los avances en el desarrollo de habilidades cognitivas y competencias comunicativas.

Los problemas matemáticos también hacen parte de la expresión simbólica, en este caso Diana Paola Avella Tuta, Fabio Ahirton Salazar Pérez y José Edilberto Miguez García de la Institución Educativa técnica Carlos Alberto Olano Valderrama, presentan resultados parciales del proyecto investigativo Resolución de problemas matemáticos con fracciones enfocados al contexto escolar, centrándose en el tema de la enseñanza de la resolución de problemas matemáticos con fracciones. En este apartado, también se encuentra la investigación desarrollada por los profesores Alfonso Jiménez Espinosa de la Universidad Pedagógica y Tecnológica de Colombia, Hugo Hernán Bohórquez Rodríguez, Marilú Castro Torres y Rafael Antonio Puentes Blanco de la Institución Educativa Técnica Antonio Nariño, quienes presentan el proyecto investigativo: Actitudes de estudiantes de cuarto y quinto, frente al aprendizaje de las matemáticas, este pretende identificar causas que generan actitudes negativas frente al aprendizaje de las matemáticas ocasionando bajo rendimiento académico.

Como factor de rendimiento académico, encontramos además el proyecto investigativo Afectividad y rendimiento académico en elárea de lenguaje, presentado por los profesores: Omaira Milena Espinel Mesa, Graciela del Carmen Samacá 
Pesca de la Institución Técnico Ramón Ignacio Avella-Aquitania y José Gabriel Cristancho Altuzarra de la Universidad Pedagógica y Tecnológica de Colombia, quienes plantean una propuesta pedagógica para desarrollar competencias emocionales que fortalezcan el proceso de aprendizaje y contribuyan al desarrollo integral de los estudiantes. Por último, como parte de este cuaderno, encontramos a los profesores Samuel Becerra Peña, Luis Orlando Martínez Quiroga de la Institución Educativa Juana Caporal y Alejandro Bolívar Suarez de la Universidad Pedagógica y Tecnológica de Colombia, con el artículo La ortografía desde los relatos anecdóticos del abuelo, que brinda algunas estrategias en la enseñanza ortográfica de las palabras oxítonas terminadas en vocal.

Babelia: este cuaderno plantea un recorrido por diferentes formas de aprendizaje hacia la construcción del multilingüismo, sin olvidar que no solamente tenemos que pensar en las lenguas dominantes del planeta, sino reconocer a Colombia como una nación multiétnica y pluricultural.

El apartado deja ver trabajos investigativos como La lectura inferencial, una clave para potenciar la comprensión lectora desarrollado por los profesores Judy Ochoa Montaña, Sandra Liliana Mesa Cárdenas, Yaqueline Pedraza Orduz de la Institución Educativa Colegio de Sugamuxi y Edgar Orlando Caro de la Universidad pedagógica y Tecnológica de Colombia, presentan un análisis de la problemática en relación con la lectura; reflexionando acerca del quehacer educativo y la importancia de fortalecer las prácticas pedagógicas para mejorar la comprensión de lectura. Enseguida encontramos la propuesta investigativa Procesos de comprensión lectora en niños de grado cuarto y quinto de primaria presentada por las profesoras Edibelcy Soler Arias y María Cristina Escobar Alba de la Institución Técnica Agropecuaria San Rafael de rondón y Lorena Agudelo Arias de la Universidad Pedagógica y Tecnológica de Colombia tiene como propósito determinar los procesos de comprensión lectora que se ven fortalecidos a partir de la implementación de estrategias diseñadas con base en la pedagogía conceptual y la "teoría de las seis lecturas" de Miguel de Zubiría Samper.

Para finalizar, se puede ver el papel de la autocrítica en la labor pedagógica del maestro presentado en el artículo titulado Para comprender el cuento, que expone brevemente la intervención, cuyo objetivo es mejorar los procesos de comprensión de los estudiantes del Colegio Boyacá de Duitama, a través de la implementación y creación de los cuentos como herramienta didáctica.

Estos trabajos no solo representan el resultado de investigaciones en el aula, sino que son el reflejo de las diversas experiencias del maestro en nuestras instituciones educativas, una forma de compartir con la comunidad académica y el contexto social. 


\section{Referencias}

Pineau, P., Dussel, I.; Caruso, M.(2001) La escuela como máquina de educar. México: Paidós.

Gómez, L., \& Pulido, O. (2016) La pedagogía y su presente: umbrales y relaciones .Praxis \& Saber 7 ( Núm.13. 9-14 